\title{
EDITORIAL
}

\section{SCIENTIFIC INQUIRY, THE MJM, AND TOMORROW'S PHYSICIAN-SCIENTIST}

In reading through the present issue of the $M J M$ and for the first time appreciating its final composition, I was reminded of the tremendous enthusiasm I felt this time six months ago, on the eve of the Spring 1996 issue's publication, for the research that had been performed by students across the globe. This enthusiasm has been rekindled by the contributions to medical science brought forth in this Spring 1996 issue of the MJM, for which the respective authors deserve much recognition.

The $M J M$ 's extensive coverage of the medical sciences is highlighted by original studies reporting a possible genetic distinction between sporadic and familial forms of hypertrophic cardiomyopathy, the role of preoperative functional status as a significant new predictor of CABG outcome in the elderly, and some critical findings on the state of health care for homeless adolescents. These investigations are complemented by exceptional and timely reviews on cancer gene therapy and the implication of homocysteine in vascular disease, as well as by a Crossroads article presenting a unique historical account of the development and use of aspirin.

New to the $M J M$ are sections entitled Case Reports, containing clinical presentations by students, beginning in this edition with a report of neonatal cholestasis associated with prenatal exposure to valproic acid, and Student Research Symposium, publishing prize-winning abstracts from student research conferences around the world, commencing in the current issue with the First Annual Meeting of the Experimental Medicine Graduate Students' Society of McGill University. Lastly, featured in the MJM Focus of this issue is Part I of a Special Forum on Tumor Markers, containing the most up-to-date clinical reviews on prostate specific antigen (PSA) and alpha-fetoprotein (AFP), written by their discoverers. The upcoming Fall 1997 issue will complete the Forum, with similar outstanding reviews on carcinoembryonic antigen (CEA) and CA-125.

Having just completed the editing of the present issue of the $M J M$, I set out on a late-evening drive across the U.S.-Canadian border and into Vermont, in which I gradually came to see with renewed clarity the vision for the $M J M$ that had fueled the efforts of all those involved with the journal over the course of the past few years. With review articles recently contributed to the $M J M$ by several of the most prominent investigators of our time, and with recent news that the $M J M$ had been accepted for indexing on Excerpta Medica/EMBASE, that the journal had just received a stellar review by the Editor of the Annals of the Royal College of Physicians and Surgeons of Canada, and that affiliations with the MJM were being considered by major student organizations around the world, it became clear that the $M J M$ was now an institution.

This led invariably to the question: "What is it about this journal that has made it come so far, so quickly?" Clearly, the tireless efforts and intense devotion of the editors have played a vital role; yet, there was something more. It was the fact that this journal proposed to achieve something on a broader scale: to serve as a forum for all students in the health sciences, to bring their research discoveries to the forefront of international awareness. But even this alone was not enough to explain the tremendous strides that had been made, and that continue to be taken.

It was then that the most significant feature became evident: that, in an era in which spending on biomedical 
research still comprises a minute portion of the federal fiscal budget in most industrialized nations, the $M J M$ provides a rare mechanism for the exploration of new paths toward medical discovery. While it is true that many studies in the $M J M$, as in most journals, have in the past reported important findings in currently active areas of research, not infrequently the $M J M$ has published, and will continue to reveal, investigations that pose highly novel questions, research motivated by hypotheses never before tested.

A forum for the inquiry and discoveries made by tomorrow's physician-scientist is critical to ensure that the medical paradigms and ideologies of yesterday and today are continually challenged. A forum that will serve as a catalyst for advancement in both medical knowledge and patient care. A forum that is the MJM.

Neil A. Goldenberg

Editor-in-Chief

Neil A. Goldenberg is the second Editor-in-Chief of the McGill Journal of Medicine and a member of the American Medical Writers Association. He graduated from Williams College (Williamstown, MA, USA) in English in 1993 and is currently in the Class of 1998 at the McGill University Faculty of Medicine (Montreal, Quebec, Canada). He has served on the editorial staff of Genetic Engineering News (New York, NY, USA) and has gained considerable experience in tumor biology and immunology, having performed research at the Garden State Cancer Center (Newark, NJ, USA) and UMD-New Jersey Medical School (Newark, NJ, USA), as well as having been awarded two summer research fellowships at the National Institutes of Health (Bethesda, MD, USA).

Copyright (C) 1996 by MJM 